\title{
DERRAME PLEURAL E ASCITE COM O ACHADO DE LINHAS B NA ULTRASSONOGRAFIA PULMONAR EM CIRRÓTICOS
}

\author{
Pleural effusion and ascites with the finding of b-lines in pulmonary \\ ultrasonography in cirrhotic patients \\ Paulo Osni Leão Perin ${ }^{1}$, Renato Roberto Piccolo ${ }^{2}$, Letícia Araújo Tassine Penatti ${ }^{2}$, \\ Ilka de Fátima Ferreira Boin ${ }^{3}$, Áurea Maria Oliveira da Silva ${ }^{4}$, Luiz Cláudio Martins ${ }^{5}$
}

\section{RESUMO}

Objetivo: O estudo teve como objetivo buscar associação entre derrame pleural e ascite, com o achado de linhas B difusas na ultrassonografia pulmonar em pacientes cirróticos, em fila de transplante hepático. Método: O presente projeto caracteriza-se como um estudo longitudinal observacional, no qual foram avaliados 56 pacientes na fila de transplante hepático, do ambulatório de transplante do Gastrocentro - UNICAMP. Nesses pacientes, foram coletados dados gerais e relacionados à doença hepática, realizada ultrassonografia pulmonar para identificação de linhas $B$ difusas e derrame pleural, e ultrassonografia abdominal para avaliar a presença de ascite. Resultados: Dentre os 56 pacientes avaliados, foram encontrados sete pacientes com derrame pleural à ultrassonografia, representando $12,5 \%$ da amostra estudada, enquanto que os sem derrame pleural somaram 49 pacientes, $87,5 \%$ do total. Além disso, foram encontradas linhas B difusas em 27 pacientes, correspondendo a $48,21 \%$ da amostra de pacientes, e ascite em 16 deles, o que correspondeu a $28,57 \%$ do total. No grupo com derrame pleural, foram encontrados três com linhas $B$, equivalentes a $42,85 \%(p=1,00)$, e cinco pacientes com ascite, correspondentes a $71,42 \%$ dos pacientes com derrame $(p=0,016)$. Conclusão: Dentre as variáveis avaliadas neste estudo, apenas derrame pleural e ascite relacionaram-se de modo estatisticamente relevante. A presença de linhas B, por sua vez, não tem relação direta com a presença de derrame pleural.

Descritores: Transplante de Fígado; Cirrose Hepática; Ultrassonografia; Derrame Pleural; Ascite.

Institution:

${ }^{1}$ Unidade de Terapia Intensiva, Departamento de Cirurgia, Universidade Estadual de Campinas, Campinas/SP, Brasil.

${ }^{2}$ Curso de Medicina da Faculdade de Medicina da Pontifícia Universidade Católica de Campinas, Campinas/SP, Brasil.

${ }^{3}$ Unidade de Transplante Hepático, Departamento de Cirurgia, Universidade Estadual de Campinas, Campinas/SP, Brasil.

${ }^{4}$ Gastrocentro, Departamento de Cirurgia, Universidade Estadual de Campinas, Campinas/SP, Brasil.

${ }^{5}$ Departamento de Medicina Interna, Universidade Estadual de Campinas, Campinas/SP, Brasil.

\section{Correspondência:}

Paulo Leão Osni Perin

HC Unicamp, Rua Vital Brasil, 251, Cidade Universitária Zeferino

Vaz, CEP 13083-888 - Campinas/SP, Brasil.

Tel.: +55 $193521-7830$

E-mail: pauloleaoperin@gmail.com

Recebido em: 01/03/2017

Aceito em: 29/04/2017

\section{INTRODUÇÃO}

Em 2015, o Brasil ocupou o segundo lugar mundial em número absoluto de transplantes hepáticos, tendo sido realizados 1805 procedimentos. ${ }^{1}$ Esses dados apontam a importância desse procedimento no cenário médico brasileiro. Sabe-se que, dentre as indicações para transplante hepático, encontram-se doenças colestáticas crônicas, doenças hepatocelulares, doenças metabólicas, doenças vasculares, tumor primário do fígado e trauma. ${ }^{2}$ Grande parte delas cursam com cirrose hepática, assim como doenças pulmonares secundárias. Dessa forma, a avaliação funcional pulmonar torna-se relevante para o portador de doença hepática, a fim de ponderar a sua gravidade clínica. 
O prognóstico dos pacientes transplantados é multifatorial, sendo que as alterações sistêmicas secundárias à doença hepática presentes previamente à cirurgia influenciam de maneira significativa o desfecho clínico desses pacientes. ${ }^{3}$

O ultrassom de pulmão tem se popularizado na medicina devido à facilidade do método na promoção de diagnósticos e escolhas terapêuticas. A técnica, por ser pouco invasiva, pode ser realizada sem grandes restrições e com riscos mínimos. ${ }^{4} \mathrm{~A}$ análise de sinais específicos, chamados de linhas $B$, colabora com boa sensibilidade para a detecção de edema intersticial. ${ }^{5-7}$

As linhas B, também conhecidas como cauda de cometa, são linhas verticais, hiperecogênicas, que se prolongam até o final da imagem, obliterando as linhas $A$ ao passar por elas. Linhas A são originadas na reverberação do ultrassom, na parede torácica e no pulmão, criando linhas horizontais hiperecogênicas, que se repetem equidistantes até o final da imagem, de modo que as linhas A são encontradas em condições fisiológicas; já as linhas $B$ são associadas ao acúmulo de líquido no parênquima pulmonar; são encontradas, principalmente, em situações de edema intersticial pulmonar, como edema agudo de pulmão, congestão pulmonar, síndrome da angústia respiratória aguda e fibrose intersticial pulmonar. ${ }^{4,6,8}$ Estudos prévios detectaram a presença de linhas $B$ em pacientes cirróticos, porém sua causa não foi completamente elucidada. ${ }^{9}$

No paciente cirrótico, a retenção hídrica é um achado comum, sendo que suas consequências podem ser encontradas em até $50 \%$ dos pacientes, seja na forma de edema, ascite ou de derrame pleural. ${ }^{10,11}$

A ascite ocorre anualmente, em aproximadamente $10 \%$ dos pacientes cirróticos, sendo que a prevalência pode chegar a $70 \%$ em dez anos de doença. São pacientes que possuem hipertensão portal que cursa com vasodilatação da circulação esplâncnica. Essa vasodilatação explica-se por produção local de mediadores vasodilatadores, principalmente óxido nítrico, secundários ao estresse endotelial vascular pela pressão aumentada no sistema porta, que culmina com o extravasamento líquido do intravascular para a cavidade abdominal. A diminuição do volume intravascular leva à ativação do sistema reninaangiotensina-aldosterona, levando à hipervolemia e retenção hídrica.$^{12} \mathrm{O}$ derrame pleural pode ser definido como o acúmulo anormal de líquido entre as duas pleuras: a visceral e a parietal. ${ }^{13,14}$ Acredita-se que 0 derrame pleural no paciente cirrótico seja contíguo ao processo de ascite, por falhas na aponeurose diafragmática,${ }^{15,16}$ associadas à pressão intra abdominal aumentada e à pressão pleural negativa, que facilita a migração hídrica ao tórax. ${ }^{17}$ Por esse motivo, a grande maioria dos derrames pleurais no paciente hepatopata são à direita, entretanto, podem ser do lado esquerdo ou bilaterais. ${ }^{15,16,18}$

\section{OBJETIVO}

O presente trabalho visa, na população estudada, elucidar possíveis associações entre a presença de derrame pleural e ascite, comparando-os com o achado de linhas $\mathrm{B}$ difusas à ultrassonografia pulmonar.

\section{MÉTODOS}

Foi realizado um estudo longitudinal observacional, no qual foram avaliados 56 pacientes na fila de transplante hepático, do ambulatório de transplante do Gastrocentro - UNICAMP. Nesses pacientes, foram coletados dados gerais e relacionados à doença hepática. Foram feitos nesse grupo: coleta de dados gerais e ultrassonografia pulmonar - para identificação de linhas B difusas, derrame pleural e ultrassonografia abdominal para avaliar a presença de ascite.

- Coleta de dados e avaliação clínica: Foram coletadas informações gerais, como idade, sexo, peso, altura, estado geral, antecedentes pessoais e familiares.

- Realização da ultrassonografia pulmonar: Foi realizada utilizando o aparelho FUJIFILM SonoSite, Inc, modelo Titan, com transdutor curvilíneo de $6 \mathrm{MHz}$, com o paciente deitado, em maca hospitalar, com a cabeceira elevada, sem contraste de microbolhas. O exame seguiu o seguinte protocolo para obtenção de imagens: com o paciente em decúbito horizontal, dividiramse ambos hemitórax em duas regiões no sentido crânio caudal (superior e inferior) e três regiões no sentido ântero-posterior (hemiclavicular anterior, linha axilar média e linha axilar posterior ou lateral da escápula). Com o transdutor no sentido transversal, foram examinados os seis pontos das regiões formadas pela intersecção das linhas traçadas, em ambos os hemitórax. Foram descritos os achados encontrados em cada um desses pontos, de acordo com a presença ou não de linhas $B$, sua quantidade, bem como a presença de derrame pleural. O exame foi concluído com a investigação da presença ou ausência de ascite. 


\section{RESULTADOS}

Dentre os pacientes avaliados, havia um total de 43 homens e 13 mulheres, totalizando 56 pacientes. Os dados coletados foram divididos entre dois grupos: os com derrame pleural e os sem.

As características do grupo estudado estão na tabela 1.

Tabela 1 - Dados comparativos entre pacientes com e sem derrame pleural considerando variáveis de sexo, idade, peso, altura, IMC, linhas B difusas e presença de ascite. Campinas, 2017.

\begin{tabular}{ccc}
\hline & Com derrame pleural & Sem derrame pleural \\
\hline TOTAL & 7 & 49 \\
Sexo $(\mathrm{H} / \mathrm{M})$ & $05 / \mathrm{fev}$ & $38 / 11$ \\
Idade & $49.43 \pm 15.16$ & $56.80 \pm 6.33$ \\
Peso & $75.8 \pm 24.42$ & $78.68 \pm 14.24$ \\
Altura & $165.79 \pm 17.81$ & $166.80 \pm 9.58$ \\
IMC & $26.97 \pm 4.82$ & $28.48 \pm 5.94$ \\
Linhas B difusas & 3 & 24 \\
Ascite & 5 & 11 \\
\hline
\end{tabular}

Foram encontrados sete pacientes com derrame pleural à ultrassonografia, representando $12,5 \%$ da amostra estudada, enquanto que, sem derrame pleural, somaram 49 pacientes, $87,5 \%$ do total. Além disso, foram encontradas linhas B difusas em 27 pacientes, correspondendo a $48,21 \%$ da amostra de pacientes, e ascite em 16 deles, o que correspondeu a $28,57 \%$ do total. No grupo com derrame pleural, foram encontrados três com linhas $B$, equivalentes a $42,85 \%(p=1,00)$, e cinco pacientes com ascite, correspondentes a $71,42 \%$ dos pacientes com derrame $(p=0,016)$.

Para descrever o perfil da amostra, segundo as variáveis em estudo, foram feitas tabelas de frequência das variáveis categóricas (linhas B difusas, ascite e derrame pleural), com valores de frequência absoluta (n) e percentual (\%), e estatísticas descritivas das variáveis contínuas (idade, peso, IMC, tabagismo, sexo), com valores de média, desvio padrão.

Para comparação das variáveis categóricas entre os dois grupos, foi utilizado o teste Qui-Quadrado de Pearson, ou o teste exato de Fisher, na presença de valores esperados menores que cinco. Para comparação das variáveis numéricas entre os dois grupos, foi utilizado o teste de Mann-Whitney, devido à ausência de distribuição normal das variáveis.

O nível de significância adotado para os testes estatísticos foi de $5 \%$, ou seja, $\mathrm{P}<0.05$.

\section{DISCUSSÃO}

$\mathrm{Na}$ população do presente estudo, a prevalência de derrame pleural foi de $12,5 \%$ e de ascite, $28,5 \%$. Quando avaliados em conjunto, essas duas variáveis têm prevalência de $32,1 \%$ na amostra estudada. Comparando com a literatura revisada, os dados encontrados são congruentes, posto que as consequências da retenção hídrica nos candidatos a transplante hepático podem ser encontradas em até $50 \%$ dos pacientes, seja na forma de edema, ascite ou de derrame pleural. ${ }^{10,11}$ Ascite isoladamente é esperada, segundo dados bibliográficos, em $10 \%$ dos pacientes, anualmente, podendo chegar em $70 \%$ em 10 anos de seguimento. ${ }^{12} \mathrm{~A}$ prevalência de $28,5 \%$ encontrada em nosso estudo corrobora essa estatística.

O hidrotórax hepático maciço está presente em até $15 \%$ dos pacientes, segundo os dados da literatura, ${ }^{15,19}$ tendo mortalidade de até $25 \%$ em um ano após o diagnóstico. ${ }^{14} \mathrm{Em}$ nosso grupo de pacientes não houve nenhum achado de hidrotórax maciço, provavelmente pelo fato do grupo avaliado estar estável clinicamente, e por terem sido estudados em regime ambulatorial.

Quando relacionadas as variáveis derrame pleural e ascite, na amostra estudada, foi encontrada relevância estatística $(p=0,016)$. Dessa forma, a teoria mais aceita para explicar o surgimento do hidrotórax no paciente cirrótico foi corroborada em nosso trabalho, a qual relaciona defeitos diafragmáticos associados a um gradiente de pressão tóraco-abdominal.

No grupo estudado não foi encontrada relação estatística significante entre a presença de derrame pleural e de linhas B $(p=1,00)$. Dessa forma, o achado de síndrome intersticial esteve presente também em pacientes sem os achados clássicos da retenção hídrica no cirrótico. Dentre os 27 pacientes que apresentaram linhas B difusas, apenas três estavam com derrame pleural. Isso implica que, além da possível congestão pulmonar secundária à hipervolemia, o acúmulo de líquido extravascular pulmonar no paciente na fila do transplante hepático pode ter outra etiologia.

Uma possível e recente explicação sugere que tal edema intersticial pulmonar possa ser predominantemente inflamatório, tendo em vista o contexto de inflamação sistêmica apresentada no paciente cirrótico. ${ }^{20} \mathrm{~A}$ presença de linhas $B$ nesses pacientes pode ser um marcador do acometimento pulmonar resultante desse processo inflamatório. ${ }^{9}$ Essa teoria pode explicar o achado de ausência de relação estatística entre o derrame pleural e as linhas $B$. 


\section{CONCLUSÃO}

Dentre as variáveis avaliadas nesse estudo, apenas derrame pleural e ascite relacionaram-se de modo estatisticamente relevante, corroborando a hipótese de que derrame pleural e ascite têm a mesma origem etiológica, de acordo com mecanismos já abordados no presente estudo.
A presença de linhas $B$, por sua vez, não tem relação direta com a presença de derrame pleural, de modo que o edema intersticial, representado pelas linhas $B$ à ultrassonografia pulmonar, pode ter outra etiologia que não a hipervolemia responsável pelo derrame pleural.

\section{ABSTRACT}

Purpose: The aim of this research is to look for an association between pleural effusion, ascites and B lines at lung ultrasound in cirrhotic patients listed for liver transplantation. Method: This is a single-center observational longitudinal research that evaluated 56 patients listed for liver transplantation, from Gastrocentro - UNICAMP. Information about general data and liver disease was gathered and the patients were submitted for lung and abdominal ultrasound for the detection of B lines, pleural effusion and ascites. Results: In the 56 patients studied, 7 of them had pleural effusion representing $12,5 \%$ of the total group and 49 of them had no effusion, $87,5 \%$ of the total. Diffuse B lines were found in 27 patients, representing $48,21 \%$ of the sample and ascites were found in 16 , corresponding $28,57 \%$ of the group. In patients with pleural effusion, B lines were detected in 3 subjects, equivalent of $42,85 \%(p=1,00)$, and ascites were found in 5 patients, representing $71,42 \%$ of the group with pleural effusion $(p=0,016)$. Conclusion: Among the variables evaluated in this study, only pleural effusion and ascites were statistically relevant. The presence of $B$ lines, on the other hand, is not directly related to the presence of pleural effusion.

Keywords: Liver Transplantation; Liver Cirrhosis; Ultrasonography; Pleural Effusion; Ascites.

\section{REFERÊNCIAS}

1. Dimensionamento dos transplantes no Brasil e em cada estado. ABTO. Ano XXII, n.4, 2016.

2. Dunn W, Jamil LH, Brown LS, Wiesner RH, Kim WR, Menon $\mathrm{KV}$, et al. MELD Accurately Predicts Mortality in Patients With Alcoholic Hepatitis. Hepatology, 2005 Feb;41(2):353-8

3. Silva JR, O de C, Sankarankutty AK, Oliveira GR, Pacheco E, Ramalho FS, Sasso KD et al. Transplante de fígado: indicação e sobrevida. Acta Cir Bras -2002;17(3):83.

4. Lichtenstein, DA. Lung ultrasound in the critically ill. Ann Intensive Care, 2014 Jan 9;4(1):1. Apud: Lichtenstein, D. A. Whole Body Ultrasonography in the Critically III. 2010.

5. Via G, Storti E, Gulati G, Neri L, Mojoli F, Braschi A. Lung ultrasound in the ICU: from diagnostic instrument to respiratory monitoring tool. Minerva Anestesiol 2012;78:1282-96.
6. Dietrich CF, Mathis G, Blaivas M, Volpicelli G, Siebel A, Wastl $\mathrm{D}$, et al. Lung $\mathrm{B}$-line artefacts and their use. J Thorac Dis. 2016; 8(6):1356-65.

7. Mohanty K, Blackwell J, Egan T, Muller M. Characterization of the lung parenchyma using ultrasound multiple scattering. Ultrasound Med Biol. 2017 May;43(5):993-1003.

8. Enghard P, Rademacher S, Nee J, Hasper D, Engert $U$, Jörres A, et al. Simplified lung ultrasound protocol shows excellent prediction of extravascular lung water in ventilated intensive care patients. Crit Care. 2015 Feb 6;19:36.

9. Perin P, Boin I, Silva A, Neto F, Martins L. Lung Ultrasound and Pulmonary Function Test in Cirrhotic Patients. Transplant Proc. 2017 May;49(4):824-8 
10. Singh A, Bajwa A, Shujaat A. Evidence-based review of the management of hepatic hydrothorax. Respiration. 2013;86:155-73.

11. Porce J. Management of refractory hepatic hydrothorax. Curr Opin Pulm Med. 2014;20:352-7.

12. Bureau C, Adebayo D, Chalret de Rieu M, Elkrief L, Valla D, Peck-Radosavljevic $M$, et al. Alfapump® system vs. large volume paracentesis for refractory ascites: A multicenter randomized controlled study. J Hepatol. 2017 Nov;67(5):940-9

13. Bhatnagar R, Maskell N. The Modern Diagnosis And Management Of Pleural Effusions. Bmj. 2015;351:H4520.

14. Walker S, Morley A, Stadon L, De Fonseka D, Arnold DT, Medford AR, et al. Nonmalignant pleural effusions: A prospective study of 356 consecutive unselected patients. Chest. 2017 May;151:1099.

15. Kashani A, Landaverde C, Medici V, Rossaro L. Fluid retention in cirrhosis: pathophysiology and management. QJM. 2008 Feb 1;(101) (Issue 2):71-85.
16. Mercky P, Sakr L, Heyries L, Lagrange X, Sahel J, Dutau $H$. Use of a tunneled pleural catheter for the management of refractory hepatic hydrothorax: a new therapeutic option. Respiration. 2010;80:348-52.

17. Kiafar C, Gilani N. Hepatic hydrothorax: Current concepts of pathophysiology and treatment options. Ann Hepatol. 2008 Oct-Dec;7(4):313-20.

18. Gurung P, Goldblatt M, Huggins JT, Doelken P, Nietert $P$, Sahn S. Pleural fluid analysis and radiographic, sonographic, and echocardiographic characteristics of hepatic hydrothorax. Chest. 2011;140:448-53.

19. Haasa K, Chen A. Indwelling tunneled pleural catheters for the management of hepatic hydrothorax. Curr Opin Pulm Med. 2017,23:351-6.

20. Dirchwolf M, Ruf A. Role of systemic inflammation in cirrhosis: From pathogenesis to prognosis. World J Hepatol. 2015 Aug 8;7(16):1974-81. 